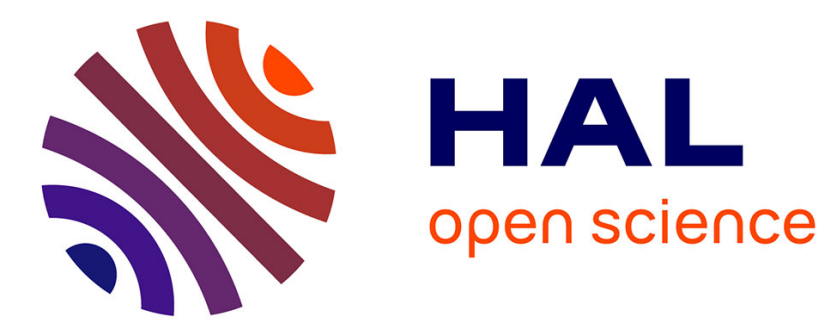

\title{
A conditional dependence approach to CO2-energy price relationships
}

\author{
Julien Chevallier, Duc Khuong Nguyen, Juan Carlos Reboredo
}

\section{To cite this version:}

Julien Chevallier, Duc Khuong Nguyen, Juan Carlos Reboredo. A conditional dependence approach to CO2-energy price relationships. Energy Economics, 2019, 81, pp.812 - 821. 10.1016/j.eneco.2019.05.010 . hal-03486986

\section{HAL Id: hal-03486986 \\ https://hal.science/hal-03486986}

Submitted on 20 Dec 2021

HAL is a multi-disciplinary open access archive for the deposit and dissemination of scientific research documents, whether they are published or not. The documents may come from teaching and research institutions in France or abroad, or from public or private research centers.
L'archive ouverte pluridisciplinaire HAL, est destinée au dépôt et à la diffusion de documents scientifiques de niveau recherche, publiés ou non, émanant des établissements d'enseignement et de recherche français ou étrangers, des laboratoires publics ou privés.

\section{다)(1) $(5$}

Distributed under a Creative Commons Attribution - NonCommerciall 4.0 International 
Version of Record: https://www.sciencedirect.com/science/article/pii/S0140988319301550

Manuscript_940b7bd1b9cc36a908d0ed4fc80ceacd

\title{
A Conditional Dependence Approach to $\mathrm{CO}_{2}$-Energy Price Relationships
}

\author{
Julien Chevallier, Duc Khuong Nguyen†, Juan Carlos Reboredo ${ }^{\ddagger}$ \\ 2nd Revision May 2019
}

\begin{abstract}
This paper uses the conditional vine copula approach to model the dependence structure between European-based carbon allowances and major energy prices. It makes two central contributions to the related literature. First, we extend the previous works of Reboredo $(2013,2014)$ by allowing for complete coverage of energy markets including natural gas, coal, and electricity, beyond the carbon-oil dependencies. Second, we simultaneously investigate the multivariate dependence among all variables in the system so that each of them can interact with the others based on a rich variety of bivariate copula functions. The consideration of the electricity market in this context offers the possibility to gauge its influences through the computation of the fuel-switching mechanism. We mainly find that there is a reliable and positive link between coal and gas prices, and between coal and oil prices, with or without the presence of electricity prices, while a weak and positive link is detected between Brent and gas prices. Carbon prices co-move only weakly with energy prices, and their link to oil and gas prices is negative. Moreover, the switch from coal to gas does not occur when the relative price of fuels taking into account carbon costs is assessed. This happens because the fuel-switching mechanism is still more costly than carbon abatement. Our findings remain intact when alternative electricity prices are used.
\end{abstract}

JEL Classification: C44; L94; Q40

Keywords: carbon price; energy markets; fuel-switching; vine copula.

*IPAG Lab, IPAG Business School, Paris, France and University Paris 8 (LED). Email: julien.chevallier@ipag.fr

${ }^{\dagger}$ IPAG Lab, IPAG Business School, Paris, France. Email: duc.nguyen@ipag.fr

${ }_{\ddagger}^{\ddagger}$ Department of Economics, Universidade de Santiago de Compostela, Santiago de Compostela, Spain. Email: juancarlos.reboredo@usc.es 


\section{Introduction}

Establishing the stochastic relationships intertwined in carbon and energy markets remains a challenging task as far as it is related to the climate change targets of carbon emission reductions, carbon efficiency of power generation, transition to renewable energy, and optimal energy investments (e.g., Ding et al., 2016; Bonneuil and Boucekkine, 2016; Zhang and Chen, 2017). Previous contributions in the field, notably Aatola et al. (2013), have generally discovered a strong link between the fundamentals (i.e., electricity, gas and coal prices) and the price of European Union Allowances (EUA). More importantly, the EUA forward prices tend to fluctuate closely with fundamentals, particularly with the price of electricity and also the gas-coal difference. Hammoudeh et al. (2014) have taken a closer look at the short-run dynamics by using a Bayesian Structural VAR. Their structural model allows us to identify that a positive shock affecting crude oil prices generates an initial positive impact on the $\mathrm{CO}_{2}$ allowance prices. Regarding gas and coal, an unexpected increase in the natural gas prices tends to reduce the price of $\mathrm{CO}_{2}$ emissions, whereas coal prices affect carbon prices only when the electricity price is omitted. Ji, Zhang and Geng (2018) study information linkages and spillover among the carbon-energy system. Their return and volatility connectedness features that crude oil, clean energy, and coal play a pivotal role. Their network confirms that the electricity market is the main net information receiver affected by the carbon market. This dynamic spillover approach has been applied recently as well by Zhang and Broadstock (2018) in commodity markets against the background of the financial crisis and also by Zhang (2017) for the oil-stock relationship.

Whenever potential interactions are at stake, several methodological frameworks (such as time series and neural networks) can be used to detect them. In recent contributions, Lutz et al. (2013) made use of the regime-switching GARCH model to investigate the nonlinear relation between the carbon price and its fundamentals including energy prices, macroeconomic risk factors and weather conditions. Their findings indicate that gas, coal, and oil can be deemed to be reliable price drivers of the $\mathrm{CO}_{2}$ price and that their influence depends on high- versus low-volatility regimes. Koch (2014) adopts a smooth transition approach to model the conditional correlations between carbon and energy prices and finds much closer carbon-energy price linkages during 2008-2012 (when compared with 2005-2007). These conditional correlations are further dependent on market uncertainty conditions. Previous 
empirical findings have in common a high degree of substitution among the three primary energy sources (i.e., crude oil, natural gas, and coal). Another relevant analytical framework is the dependence-switching CoVaR-copula model, applied by Ji, Liu, Nehler, and Salah Uddin (2018), to (clean) energy returns and uncertainty changes; by Ji, Bouri, Roubaud, and Shahzad (2018) to energy and agricultural commodities; and by Kumar et al. (2018) to the BRICS stock and foreign exchange markets. In these empirical applications, tail dependence for different market conditions can be indeed estimated using a dependence-switching copula model.

In this paper, we further extend the investigation of the cross-relationships between the $\mathrm{CO}_{2}$ price and energy markets by advancing two central contributions. The first contribution deals with the choice of data. We decided to work not only with $\mathrm{CO}_{2}$, oil, gas and coal but also with the price of electricity. The rationale behind this idea is that variations in electricity prices have significant implications on the choice of power plants and fuels, which in turn affect carbon emissions and their prices. Meanwhile, the fluctuations in carbon prices can change the merit order of coal and gas plants whereby the efficiency of the plants and the fuel costs are critical decision factors. The exact relationship between energy and carbon prices could thus be biased if the dynamics of electricity prices is ignored. Here, the influence of the electricity variable is captured through the construction of a specific spread, which we termed the 'fuel-switching price'. Delarue and D'haeseleer (2007) defined it as the $\mathrm{CO}_{2}$ price at which a gas-fired power plant is as economically and equally profitable as a coal-fired power plant. It exists for every pair of gas and coal plants. The fuel-switching price thus brings meaningful content for energy economists and policymakers. However, its influence has been omitted in most of the previous literature, except in engineering journals (see Delarue et al., 2010).

The second contribution is a methodological one because we capture the dependence structure by resorting to the vine copula approach. This methodological framework offers the advantage of modeling various dependence structures and also their potential to construct a rich set of distributions, in the spirit of Reboredo $(2013,2014) .{ }^{1}$ We go one step further than these previous works by examining the dependence structure between a more extensive

\footnotetext{
${ }^{1}$ It is worth noting that we are not leaning towards establishing causal relationships in this paper. The very logic of copula is instead to study dependencies; that is, how are markets linked in average and extreme market conditions, with a specific focus on the moderating role played by the electricity variables.
} 
array of time series in the carbon and energy spheres from a multivariate setting perspective.

The objective of this paper is to develop a time series model including the fuel-switching price that allows for various interesting applications for economists, as suggested by Zachmann (2013). Indeed, to the extent that multi-commodity options are quite common in electricity markets, the ability of the stochastic model to statistically capture the commodity co-movements would help assess the effectiveness of these options. The results from our vine copula analysis mainly show a complex dependence structure between the price variables under consideration. In particular, we find a strong dependence between the fuel-switching price, coal and gas prices. The dependence is positive and high between coal prices, and the switch variable with symmetric upper and lower tail dependence; whereas it is harmful and high between gas prices and the switch variable. We also find that $\mathrm{CO}_{2}$ prices weakly co-move with energy prices, especially with the switch variable (as a proxy for electricity prices) that displays low and negative dependence. Overall, high carbon costs do not appear as a sufficient criterion to trigger the switch from coal- to gas-fired plants (potentially owing to the engineering costs accrued by shut down/power up installations in the process).

The rest of this paper is structured as follows. Section 2 provides a brief review of the inter-relationships between energy and carbon markets. Section 3 presents the data. Section 4 describes the methodology. Section 5 contains the empirical results. Finally, Section 6 summarizes the main findings.

\section{Power sector and $\mathrm{CO}_{2}$ pricing}

In the power sector, the marginal generation technology is essential to the determination of electricity prices. $^{2}$ There is thus 'merit order' which ranks the different generation units by marginal costs from the cheapest technology to the most expensive. Sijm et al. (2005) show that several parameters including fuel prices, plant efficiencies, carbon intensity, and carbon costs are critical for this ranking. Likewise, the merit order of power plants may change with the introduction of carbon costs through climate policy instruments.

In the following, we build on the notations developed by Delarue et al. (2008) who

\footnotetext{
${ }^{2}$ In the electricity price forecasting literature, electricity prices are either taken as they are or transformed with functions which can handle negative prices (such as probability integral transform), see e.g., Uniejewski, Weron and Ziel (2018).
} 
derived the E-Simulate model at the KU Leuven university in Belgium. ${ }^{3}$ This is a European simulation model of interconnected power system networks that are developed according to the physical characteristics of the electricity grid.

Assuming no carbon costs, the marginal cost of electricity is represented by the ratio between fuel costs and the plants' efficiencies, such as:

$$
M C=\frac{F C}{\eta}
$$

where $M C$ is the marginal cost, $F C$ is the fuel cost, and $\eta$ is the plant's efficiency. ${ }^{4}$

When the carbon costs are introduced, the marginal cost for each plant accounts for an emissions factor which typically depends on the fuel and the quantity of fuel burnt:

$$
M C=\frac{F C}{\eta}+\frac{E F}{\eta} E C
$$

where $E F$ is the emissions factor and $E C$ is the emissions costs. The switching point between a given coal plant and a given gas plant can be defined as the emissions cost that equalizes marginal costs; that is, $M C_{g a s}=M C_{\text {coal }}$. This represents the allowance cost that leads to a switch between two plants in the merit order. This price interacts with various factors related to fuel prices, energy efficiency, and associated $\mathrm{CO}_{2}$ emissions:

$$
E C_{\text {switch }}=\frac{\eta_{\text {coal }} F C_{\text {gas }}-\eta_{\text {gas }} F C_{\text {coal }}}{\eta_{\text {gas }} E F_{\text {coal }}-\eta_{\text {coal }} E F_{\text {gas }}}
$$

If the carbon price is higher than this cost, then generating electricity from gas is more profitable than from coal. Besides, the allowance cost of the switching point varies linearly with the coal-to-gas price ratio. Consider the fuel price ratio $r=\frac{F C_{\text {gas }}}{F C_{\text {coal }}}$, then write the so-called switching point:

$$
E C_{\text {switch }}=\frac{\left(\eta_{\text {coal }} r-\eta_{\text {gas }}\right) F C_{\text {coal }}}{\eta_{\text {gas }} E F_{\text {coal }}-\eta_{\text {coal }} E F_{\text {gas }}}=a r-b
$$

with $a=\frac{\eta_{\text {coal }} F C_{\text {coal }}}{\eta_{\text {gas }} E F_{\text {coal }}-\eta_{\text {coal }} E F_{\text {gas }}}$ and $b=\frac{\eta_{\text {gas }} F C_{\text {coal }}}{\eta_{\text {gas }} E F_{\text {coal }}-\eta_{\text {coal }} E F_{\text {gas }}}$. Eq.(4) shows that the switching

\footnotetext{
${ }^{3}$ The notations developed in this section draw substantially on previous works by Chevallier (2011) and Chevallier and Goutte (2017).

${ }^{4}$ In this paper, we do not consider the costs associated with regulatory changes, workforce, and operations and maintenance. This assumption allows us to make the model more inclined to empirical work.
} 
point depends linearly on the fuel price ratio. It also shows that switching can occur even with zero allowance costs. The denominator is positive if $\frac{\eta_{g a s}}{\eta_{\text {coal }}}>\frac{E F_{\text {gas }}}{E F_{\text {coal }}}$. This relationship is verified in practice: the emissions factor for gas-fired power plants is nearly half of that for coal-fired power plants. Additionally, the efficiency of gas-fired power plants (especially CCGT) is usually higher than that for coal-fired power plants.

If $r>\frac{\eta_{\text {gas }}}{\eta_{\text {coal }}}$, then $E C_{\text {switch }}>0$. It means that the carbon cost for switching is positive. On the contrary, it is interesting to notice that fuel-switching could even occur will null carbon costs. Therefore, for a given $(>0)$ coal price, it is theoretically possible to have a gas price that is low enough to influence the power producers' fuel-switching behavior, even with null carbon costs (whenever the coal-to-gas ratio is equal to the ratio of the gas and coal efficiencies, see e.g. Delarue et al., 2009)).

The introduction of $\mathrm{CO}_{2}$ costs provides incentives for generators to use gas instead of coal at the switching point. Depending on the load and the number of gas units available, the emissions cost profile differs.

In practice, the main abatement opportunities occur by switching from coal to gas. Notice switching from/to oil is limited in Europe. Similarly, switching from/to nuclear is difficult because nuclear energy is not flexible.

To compute the switch price, we plug in the values cost $_{\text {ngas }}=0.5$ as the production cost of one MWh of electricity based on net $\mathrm{CO}_{2}$ emissions of gas (e.g. the efficiency of the typical gas plant, expressed in EUR/MWh), cost $_{\text {coal }}=0.4$ the production cost of one MWh of electricity based on net $\mathrm{CO}_{2}$ emissions of coal (e.g. the efficiency of the typical coal plant, expressed in EUR/MWh), $t C O 2_{\text {coal }}=364.68$ the emissions factor (expressed in $\mathrm{kgCO}_{2} \mathrm{eq} / \mathrm{MWhp}$ ) of a conventional coal-fired plant, and $t C O 2_{\text {ngas }}=210.96$ as the emissions factor (expressed in $\mathrm{kgCO}_{2} \mathrm{eq} / \mathrm{MWhp}$ ) of a conventional gas-fired plant (see Delarue and D'haeseleer (2007, 2008), Delarue et al. (2008)).

Technical values for typical coal- or gas-fired power installations are required for the computation of the switch price, which is a theoretical (unobserved) price that needs to be computed for this study. The references provided above are taken from recognized engineering journals regarding the calibration of the E-Simulate model at the K.U. Leuven in Belgium, which was used extensively to model the electricity grid in Europe. Several scholars (see, e.g., Convery and Redmond, 2007) or even the EU DG Clima have re-used their estimates in several reports. 
As long as the carbon price lies below this switching price, coal plants are more profitable than gas plants - even after taking carbon costs into account.

In the next section, we will examine in more detail the effects of EUA prices, on the one hand, energy and power prices, on the other hand.

\section{Data}

\subsection{Data description}

Our dataset consists of the ICE ECX EUA Daily Futures - SETT. PRICE (in Euro per ton of $\mathrm{CO}_{2}$ ), the Crude Oil-Brent Cur. Month FOB U\$/BBL, the ICE Natural Gas 1 Mth.Fwd. P/Therm, the Coal ICE API2 CIF ARA Nr Mth \$/MT - SETT.PRICE, and the fuel-switching price; computation is detailed in Section 2. Energy prices are pictured in Figure 1.

We obtained 1598 daily prices that spanned the period from 1 January 2010 to 19 May 2016. All price series were converted to US dollars using the bilateral exchange rates given by the European Central Bank and the Federal Reserve Bank of St. Louis. From daily prices, including the fuel-switching price, we computed the price returns at each time $t$ as $\left(p_{t}-p_{t-1}\right) / p_{t-1}$, where $p_{t}$ denotes the price at time $t^{5}$.

\footnotetext{
${ }^{5}$ Notice in our paper, we are not using logarithmic returns. It is not possible given that our switch variable can take positive or negative values. Therefore, we use the percentage change of the level of series as given by $\frac{\left(p_{t}-p_{t-1}\right)}{p_{t-1}}$, where $p_{t}$ denotes the price level of the corresponding series at time $t$.
} 
Figure 1: Energy Prices
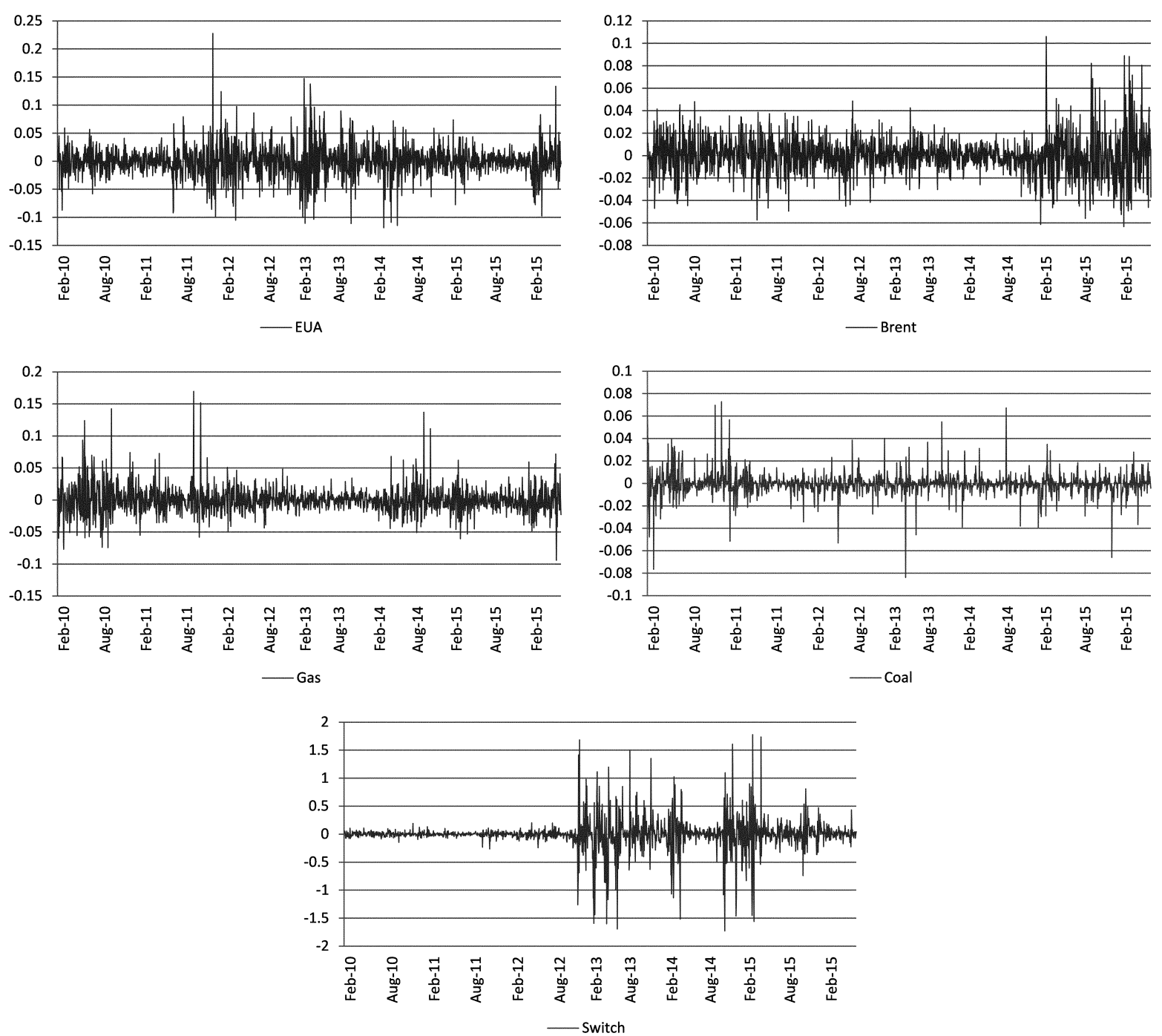


\subsection{Descriptive statistics}

Table 1 reports the descriptive statistics and the basic stochastic properties for carbon allowance price returns (EUA), oil price returns (Brent), gas price returns (Gas), coal price returns (Coal) and fuel-switching price returns (Switch). The mean returns are close to zero for all series and are relatively low concerning their standard deviations. Standard deviations and differences between maximum and minimum values show that the Switch series is much more volatile than the other series. Coal prices display lower volatility. Negative values for skewness are only observed for the Switch variable; the other variables only display positive skewness. All the returns exhibit excessive kurtosis, confirming the presence of fat tails in the marginal distributions or a relatively high probability of extreme observations. The Jarque-Bera test for normality rejects the normality of the unconditional distribution for all the series. The Ljung-Box statistic for autocorrelation up to 20th order in the returns suggests the presence of serial correlation in the Coal and Switch series and no evidence of serial correlation for the other series. Finally, the results of the Lagrange multiplier for the ARCH (ARCH-LM) statistic for serially correlated squared returns indicate that ARCH effects are likely to be found in all the return series.

\section{[Insert Table 1 here]}

Table 2 reports information on the Pearson linear correlation coefficient, with values indicating that Brent with Gas, Brent with Coal and Coal with Gas display positive dependence, Switch with Gas displays negative dependence and Switch with Coal displays positive dependence. All other pairs show very low or nonlinear dependence.

[Insert Table 2 here] 


\section{Methodology}

We study multivariate dependence between carbon allowance prices and major energy prices, considering the dependence structure between (a) $\mathrm{CO}_{2}$, Brent, natural gas and coal prices, because this helps track interactions between the $\mathrm{CO}_{2}$ market and each of the major energy markets in a multivariate and integrated setting. (b) $\mathrm{CO}_{2}$, Brent and electricity prices (Switch), because this reveals the influence of the electricity market on carbon allowance prices and the substitutability potential for crude oil with the other two energy sources (Gas \& Coal). (c) $\mathrm{CO}_{2}$, Brent, natural gas, coal and electricity prices, because these reveal interactions between carbon allowance prices and all energy prices. For multivariate dependence modeling, we employ the vine copulas briefly described below.

\subsection{Modelling multivariate dependence with vine copulas}

A copula function is a uniform multivariate distribution function of a $d$-dimensional multivariate random vector with support in $[0,1]^{d}$. According to the Sklar's (1959) theorem, the copula function $C(\cdot)$ is related to the cumulative distribution function of a random vector, $F\left(x_{1}, x_{2}, \ldots, x_{d}\right)$, via:

$$
C\left(u_{1}, u_{2}, \ldots, u_{d}\right)=F\left(x_{1}, x_{2}, \ldots, x_{d}\right),
$$

where $u_{i}=F_{i}\left(x_{i}\right)$ is the value of the marginal distribution function of the variable $x_{i}$ for $i=1, \ldots, d$. The converse of Eq. (5) is also true: any multivariate distribution function can be written using its marginal distributions and a copula function. Assuming that the marginals and copula $C$ are differentiable, then the density representation of the copula is given by

$$
f\left(x_{1}, x_{2}, \ldots, x_{d}\right)=f\left(x_{1}\right) f\left(x_{2}\right) \cdots f\left(x_{d}\right) c\left(F_{1}\left(x_{1}\right), F_{2}\left(x_{2}\right), \ldots, F_{d}\left(x_{d}\right)\right)
$$

where $f_{i}\left(x_{i}\right)$ are the marginal densities and $c(\cdot)$ is the copula density given by

$$
c\left(u_{1}, u_{2}, \ldots, u_{d}\right)=\frac{\partial^{d} C\left(u_{1}, u_{2}, \ldots, u_{d}\right)}{\partial u_{1} \partial u_{2} \cdots \partial u_{d}} .
$$

Thus, the multivariate density is represented by the product of the marginal densities and the multivariate copula density that accounts for the structure of dependence. This feature allows 
for model flexibility by untying univariate marginals distribution estimates from dependence estimates.

Although several multivariate copulas (such as the Gaussian, Student-t or Archimedean copulas) have been used to model multivariate dependence, they lack modeling flexibility for a large number of variables (e.g., by imposing the same structure of dependence for bivariate pairs). Therefore, vine copulas - which were initially proposed by Joe (1996) - have been developed to describe multivariate dependence.

Vine copulas are multivariate copulas that are generated using a hierarchical structure given by a cascade of bivariate copulas (pair copulas), where each bivariate pair copula captures the conditional dependence between two random variables. Vine copulas are obtained by decomposing the joint probability density in Eq. (6) by iterative conditioning. However, a crucial issue in this density decomposition is the choice regarding the order of the variables. The literature includes several vine specifications that are made of different pair-copula constructions composed of $d(d-1) / 2$ bivariate copulas (see, e.g., Bedford and Cooke, 2001, 2002; Kurowicka and Cooke, 2006; Aas et al., 1999). In our research, we focus on three vine models with different hierarchical tree structures that have been widely employed in the empirical literature, namely, C-vine, D-vine, and R-vine copulas. Specific details on those copulas are reported in the Appendix.

\subsection{Marginal and copula models}

The marginal densities and the bivariate copulas are the main building blocks of multivariate dependence modeled using a vine copula. The marginal models and copula specifications used in the empirical analysis are described as follows.

To account for the main characteristics of the distribution of energy price changes (such as leverage, fat tails and asymmetries), we consider that the conditional mean and variance of the returns $\left(r_{t}\right)$ are given by an autoregressive moving average (ARMA) model with $p$ and $q$ lags given by:

$$
r_{t}=\phi_{0}+\sum_{j=1}^{p} \phi_{j} r_{t-j}+\sum_{h=1}^{q} \varphi_{h} \varepsilon_{t-h}+\varepsilon_{t},
$$

where $\epsilon_{t}=\sigma_{t} z_{t}$ such that $z_{t}$ is a zero mean and the unit variance $i . i . d$. random variable that 
follows a Student-t density distribution and $\sigma_{t}^{2}$ is the conditional variance, with dynamics described by a threshold generalized autoregressive conditional heteroskedasticity (TGARCH) specification:

$$
\sigma_{t}^{2}=\omega+\sum_{k=1}^{r} \beta_{k} \sigma_{t-k}^{2}+\sum_{h=1}^{m} \alpha_{h} \varepsilon_{t-h}^{2} \sum_{h=1}^{m} \lambda_{h} 1_{t-h} \varepsilon_{t-h}^{2},
$$

where $\omega$ is a constant, $\sigma_{t-k}^{2}$ is the GARCH component, $\varepsilon_{t-h}^{2}$ is the ARCH component and $1_{t-h}$ is the indication function equal to 1 if $\varepsilon_{t-h}<0$ and otherwise equal to 0 . The parameter $\lambda$ captures asymmetric effects in such a way that when $\lambda>0$, the future conditional variance will proportionally increase more following a negative shock than following a positive shock of the same magnitude.

To model bivariate dependence, we use ten different copula specifications to capture different characteristics of dependence, such as tail independence and symmetric and asymmetric tail dependence. Table 3 summarizes the main features of all the copula functions specifications used in the empirical analysis.

\section{[Insert Table 3 here]}

Marginal and copula parameters are obtained following a two-step procedure. First obtained by maximum likelihood are the parameters for the univariate marginal models in Eq. (8)-(9) and also the standardized residuals that will be the pseudo-sample observations for the copula. ${ }^{6}$ Next obtained are the parameters of the vine copula using the sequential maximum likelihood estimation procedure, as introduced by Aas et al. (2009) and discussed in Hobaek and Haff (2013). This consists of sequentially estimating pair copula parameters and conditioning them on the parameters of the previous levels of the vine structure. Thus, given the selected tree, for the first tree level we estimate the bivariate copula parameters by maximum likelihood and choose the best pair copulas - from the family reported in Table 3 - using the Akaike information criterion, as described in Brechmann and Czado (2011) and Dißmann et al. (2012). Then, for the next tree level, we compute the pseudo-sample observations from the estimated copulas of the previous level using Eq. (11) and then obtain

\footnotetext{
${ }^{6}$ We cannot conduct robustness checks on raw data because raw returns are not i.i.d. Therefore, econometric theory teaches us to infer dependency relationships from residuals instead. This is standard practice in copula estimation (see Jaworski et al., 2010 for a review).
} 
the copula parameter estimates for this tree. This process is repeated sequentially until tree $d-1$.

\section{$5 \quad$ Results}

\subsection{Results for the marginal distribution models}

Table 4 reports the estimation results for the marginal models described in Eqs. (8)-(9). The parameters $p, q, r$ and $m$ were selected for different combinations of values ranging from zero to a maximum lag of two, using, for this purpose, the AIC values. Accordingly, an $\operatorname{ARMA}(0,0)$-TGARCH(1,1) specification was selected for all return series except the coal series, where a single lag was considered to account for serial correlation as described in Table 1. The estimation results indicate that the conditional volatility is quite persistent for all the series. Volatility asymmetric effects are observed for all the series, except coal. Furthermore, neither autocorrelation nor $\mathrm{ARCH}$ effects remained in the residuals of the marginal models.

\section{[Insert Table 4 here]}

We assessed the adequacy of the marginal distribution model by testing the null that the standardized residuals were uniform $(0,1)$. We used the Kolmogorov-Smirnov, Cramervon Mises and Anderson-Darling tests, which compare the specified theoretical distribution function with the empirical distribution function. The last three rows of Table 4 report the $p$ values for those tests, which indicate that the null of the correct specification of the distribution function cannot be rejected at the $5 \%$ threshold level. We also checked for the presence of structural breaks in the standardized residuals by using the exponential likelihood ratio F-statistic - as developed by Andrews (1993), Andrews and Ploberger (1994) and Hansen (1997) — which endogenously identifies one structural breakpoint. For all series, we were unable to reject the null of no change point in the standardized residuals. Similarly, the multiple breakpoint tests developed by Bai and Perron (1998) was unable to identify any structural change in the standardized residuals. The marginal models are, thus, accurately specified and the copula model can correctly capture the dependence structure among energy prices. 


\subsection{Results for the vine copula models}

We now discuss the empirical results for three different market situations with and without the fuel-switching price variable. This analysis offers the possibility to assess the role of the switch variable in the formation of coal prices.

CASE 1: $\mathrm{CO}_{2}$, Brent, natural gas and coal prices.

Figure 2: C-vine copula tree for Brent (B), gas $(\mathrm{G})$, coal $(\mathrm{C})$ and EUA (E).

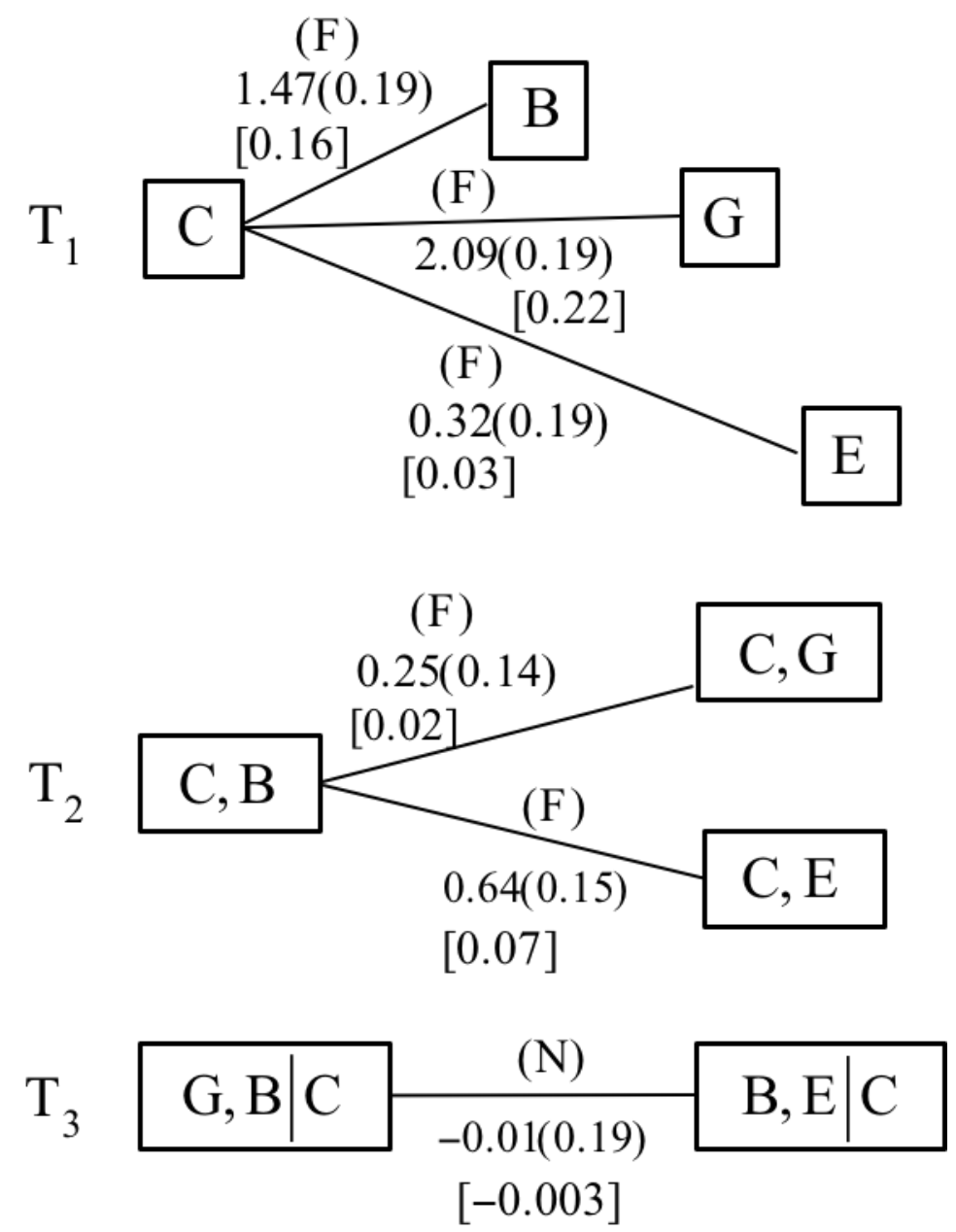

Note: For each edge, we have indicated the name of the best pair copula (see Table 3) and its estimated parameter value with the corresponding standard error (in parenthesis) and Kendall's tau (in brackets).

We first estimated the three vine copula models in Eqs. (10), (12) and (13) using the pair copulas in Table 3 for $\mathrm{CO}_{2}$, Brent, natural gas and coal prices. Table 5 reports results 
for different selection criteria; the best copula fit was achieved by the C-vine copula model represented in Figure 2, along with the corresponding information on the pair copulas. Accordingly, the dependence structure between prices for $\mathrm{CO}_{2}$ and energy is dominated by coal, which exhibits greater dependence with Brent and gas prices (with Kendall's taus of 0.16 and 0.22 , respectively) than with $\mathrm{CO}_{2}$ prices.

\section{[Insert Table 5 here]}

The EU ETS was designed to foster innovation in technologies that are less carbonintensive than coal, which is often the cheapest alternative for power production. The introduction of carbon costs is meant to integrate the social value of carbon into the business models of user companies. We can observe, with this first set of results, that coal is the benchmark polluting technology and that it is difficult to trigger a move towards a lowcarbon era.

Moreover, Figure 2 reveals that conditional dependence between Brent and gas prices and Brent and $\mathrm{CO}_{2}$ prices is weak; this is also the case for conditional dependence between gas and $\mathrm{CO}_{2}$ prices. The best pair copulas reveal that there is no upper or lower tail dependence. This result is consistent with the fact that spikes in energy prices are independent and so are not transmitted between different energy sources. Overall, our evidence indicates that coal, oil, and gas prices are, on average, integrated, even though they behave as if independent in the case of extreme upwards or downwards movements; in contrast, $\mathrm{CO}_{2}$ prices co-move weakly with different energy prices.

We now investigate in more depth whether inclusion of the electricity market (switch variable) will reveal any new interplays between $\mathrm{CO}_{2}$ and the energy markets.

\section{CASE 2: $\mathrm{CO}_{2}$, Brent and electricity prices (switch).}

Different selection criteria show that there is no difference between the three vine copula structures. Figure 3 depicts a similar structure for the three models. The results for multivariate dependence between $\mathrm{CO}_{2}$, Brent and electricity prices for the three vine copula models are presented in Table 6.

[Insert Table 6 here] 
Figure 3: D-vine copula tree for Brent (B), switch (S) and EUA (E).

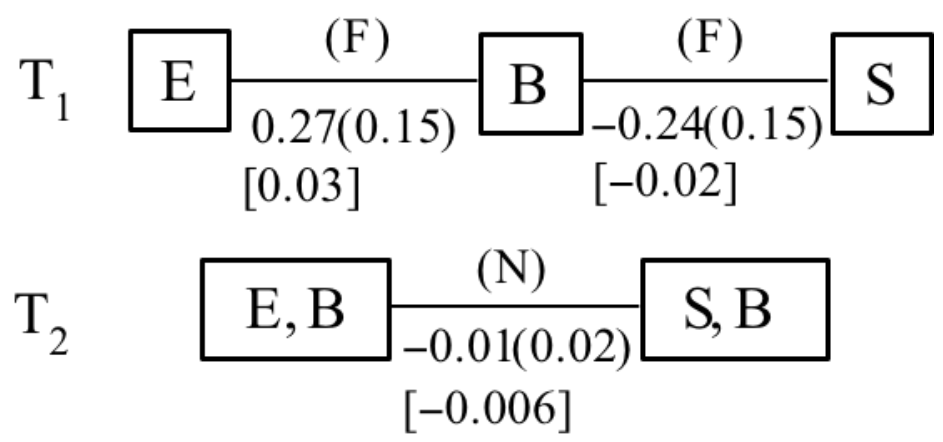

Note: For each edge, we have indicated the name of the best pair copula (see Table 3) and its estimated parameter value with the corresponding standard error (in parenthesis) and Kendall's tau (in brackets).

Estimation of the multivariate dependence structure indicates that there is weak dependence between $\mathrm{CO}_{2}$ and Brent, and also between Brent and switch, as Kendall's tau takes low values (0.03 and -0.02, respectively). Likewise, the dependence between $\mathrm{CO}_{2}$ and switch as given by the Gaussian copula is extremely weak. For all the pairs, we find evidence of no tail dependence, indicating that these prices are always independent of extreme upward or downward energy price movements.

This second set of results indicates that power companies did not immediately introduce carbon cost pricing into their usual dark and spark spreads. At the time of initial allocation of $\mathrm{CO}_{2}$ allowances, these were free and, given their price evolution until the early phase of the financial crisis launched in 2008, there were insufficient incentives to reduce coal use and increase gas use, even though there were moments when gas was cheaper than coal. To ensure profitability, the power company needs a sufficiently significant and stable price gap between the two fuels for several weeks or months, after accounting for all costs related to the switch between fuels (engineers need several days to close down a facility and open a new one). In Phase III, we would expect the expectations of these companies to change dramatically with the move to auctioning (i.e., with the need to bid for each $\mathrm{CO}_{2}$ allowance). This increases the burden of $\mathrm{CO}_{2}$ costs and adds to the fuel-switching formula (clean spark and explicit dark spreads). 
CASE 3: CO2, Brent, natural gas, coal and electricity prices.

\section{[Insert Table 7 here]}

In considering $\mathrm{CO}_{2}$ prices simultaneously with the four energy prices, the results of the selection criteria for multivariate dependence reported in Table 7 indicate that - based on an analysis of their tree hierarchical structures - the R- and D- vine copula structures yield similar results and outperform the C-vine structure.

Figure 4: D-vine copula tree for Brent (B), gas (G), coal (C), switch (S) and EUA (E).

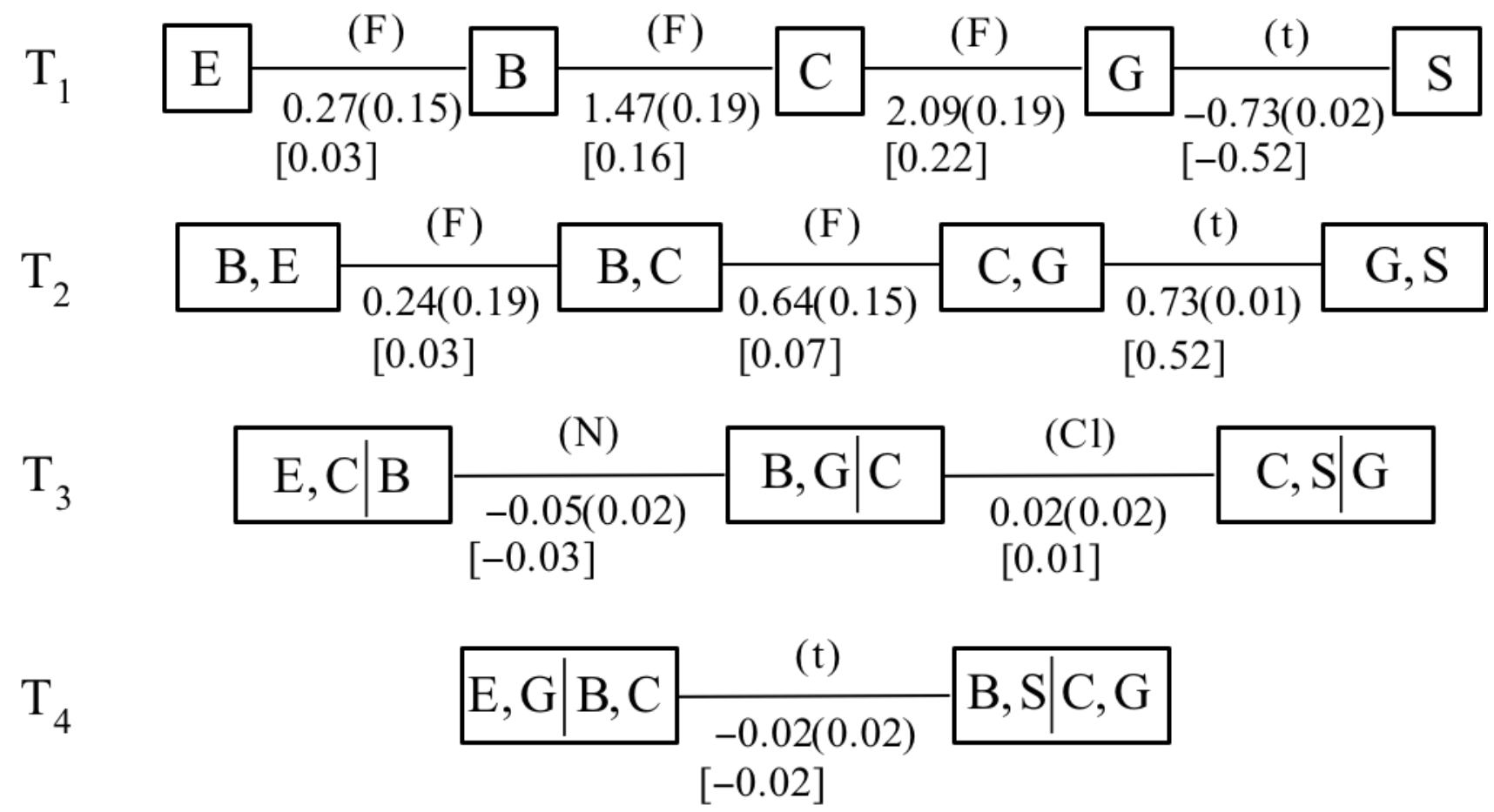

Note: For each edge, we have indicated the name of the best pair copula (see Table 3) and its estimated parameter value with the corresponding standard error (in parenthesis) and Kendall's tau (in brackets).

Figure 4 represents this dependence structure, along with information on the pair copulas. Pair dependence in the first tree indicates the existence of positive and significant dependence between Brent and coal prices, and also between gas and coal prices. There is also strong 
negative dependence between the gas and switch variables, with Kendall's tau of -0.52, and positive dependence between Brent and $\mathrm{CO}_{2}$ prices is weak, with Kendall's tau of 0.03.

In the second tree of the multivariate dependence structure, there is a positive dependence between Brent and gas prices and there is also strong positive conditional dependence (Kendall's tau of 0.52) between the coal and switch variables. The latter finding is also consistent with evidence of symmetric upper and lower tail dependence, given that the Student-t copula is the best model in describing the coal-switch dependence structure. In contrast, there is weak dependence between coal and $\mathrm{CO}_{2}$ prices (Kendall's tau of 0.03) and between the Brent and natural gas prices (Kendall's tau of 0.07). The third tree reveals weak conditional dependence between gas and $\mathrm{CO}_{2}$ prices, and also weak dependence between Brent prices and the switch variable. Finally, the fourth tree indicates that conditional dependence between $\mathrm{CO}_{2}$ prices and the switch variable is very weak and negative, as indicated by an associated Kendall's tau of -0.02 .

Overall, when all energy prices are included in the multivariate dependence analysis, the primary evidence provided by different vine copula model structures can be summarized as follows. First, there is a strong dependence between electricity prices, as measured by the switch variable, and coal and gas prices. Dependence is, however, positive and high between coal prices and the switch variable, with symmetric upper and lower tail dependence, whereas dependence is negative and high between gas prices and the switch variable. Second, there is a strong positive dependence between coal and gas prices and between coal and Brent prices, which may reflect concomitant increases in energy prices in economic boom periods. Third, $\mathrm{CO}_{2}$ prices weakly co-move with energy prices, with negative dependence on Brent and gas prices. Finally, $\mathrm{CO}_{2}$ prices are weakly and negatively associated with the switch variable, suggesting that high carbon costs will not activate the switch from coal-fired to gas-fired plants.

It is worth noting that the finding of a positive link between coal prices and the switch variable in the first case described in the previous paragraph typically suggests that energy producers, to produce electricity, tend to shift between energy sources as an input at the installation level. In practice, the switch from coal to gas occurs when relative prices make gas (cheaper as a baseline fuel) more profitable than coal as an input for electricity production at the installation level, after taking into account carbon costs. Inversely, an increase in gas prices will prevent the switch from gas to coal plants when assessing the relative price of 
fuels (including carbon costs). In this case, electricity producers would keep operating the installation with the baseline technology.

Finally, the nature of dependence among energy and carbon emissions prices does not differ with and without electricity prices since their relationships are mostly described by the same symmetric copulas (Frank, Gaussian, and Student-t copulas). The exceptions include the relationship between electricity prices on the one hand, and natural gas and coal prices, on the other hand. The robustness of our dependence results was checked by considering potential structural breaks in market dependence or time-varying dependence. Concretely, the dependence analysis was performed by splitting the sample period into two subsamples (January 2010 to July 2013 and August 2013 to May 2016) and considering time-varying parameter pair copulas. The empirical evidence from the structural break analysis fully confirms the dependence results reported for the whole sample period and by the reported static copulas. ${ }^{7}$

\subsection{Robustness}

We have checked the robustness of our results by considering daily spot prices form EPEX spot for Germany/Austria instead of our switch variable ${ }^{8}$. Indeed, the main empirical findings show that for cases 2 and 3 (note that case 1 does not focus on power prices) our evidence on dependence remains similar. We typically find that hierarchical dependence in case 2 for EUA, Brent and power prices remained identical and with similar bivariate dependence structures. Similarly, for case 3 among EUA, Brent, coal, gas and power prices the hierarchical dependence changed from a D-vine to a C-vine, even though bivariate dependence results point to similar evidence than the one we reported for the switch variable. Overall, analysis with EPEX spot prices is entirely consistent with the evidence we obtain by using the switch variable.

\footnotetext{
${ }^{7}$ For reasons of brevity, results for the structural break test and for time-varying pair copulas are not reported here, but are available on request.

${ }^{8}$ The detailed results are not reported here for the sake of concision but can be entirely made available upon request to the authors. We wish to thank an anonymous reviewer for this valuable suggestion.
} 


\subsection{Discussion}

If we look at the big picture - that is, how the obtained results can be used in the current debate on $\mathrm{CO}_{2}$ emissions - , then we need to reflect on the experience gathered with carbon and GHG trading systems, while focusing on the performance of these mechanisms as they have been implemented in practice.

The United Nations Framework Convention on Climate Change (UNFCCC) clearly states that greenhouse-gas concentrations in the atmosphere coming from anthropogenic interference need to be stabilized as much as possible to prevent climate change to a 2 degrees Celsius scenario at the 2050 to 2100 horizon (see Hoffert et al., 1998).

At the European level, several scholars have analyzed the contribution of the EU ETS to the management of $\mathrm{CO}_{2}$ emissions with reductions, if any, coming from abatement at the cheapest sources of pollution. Based on early 2005-2007 data, Ellerman and Buchner (2007) have documented design flaws giving rise to possible over-allocation in the system. The scheme has also been affected by erratic (low) allowance price movements in the wake of inter-Phases I and II banking restrictions (see, e.g., Alberola and Chevallier, 2009).

In this paper, based on 2010-2016 European data, we contribute to this debate by empirically establishing that high $\mathrm{CO}_{2}$ prices alone are insufficient to push the electricity producers to switch from coal-fired plants to gas-fired plants. In practice, this means that the emissions trading scheme is not functioning at its maximum potential to provide incentives for polluters to switch to the lower carbon-intensive fuel (gas). This happens because of the price differential between coal and gas that is not compensated enough by the introduction of carbon costs in the range of 20 to 30 euros per ton of $\mathrm{CO}_{2}$-equivalent. Our central policy implication for the GHG emissions debate is that the tutelary value of carbon should be much higher than the current historical means. Perhaps, the $\mathrm{CO}_{2}$ price should oscillate in the range of a hundred euros per ton to effectively foster the switch away from coal pollution towards gas-fired power installations.

Concerning the industry operations management, we can advance another practical implication of our paper. If the relative price differential between gas and coal (including carbon costs) is sufficient to trigger the fuel-switching mechanism, then practically this task requires manual engineering work. That is to say, a team of engineers and technical assistants needs to manually operate the factory to shut down a coal-fired installation and redirect power production towards a gas-fired installation. The whole process could take over a day and 
a half to three days before the factory can be considered as shut down. Therefore, we uncover in this paper that in reality, the fuel switching mechanism occurs on fewer occasions than economists initially believed it would in theory (fostering the targeted carbon emissions abatement). In a nutshell, it is because the price differential (including a massive carbon cost, most likely higher than the 35 Euro historical high) must be prolonged for several days (ideally a week or two) before it becomes profitable actually to shut down a factory and open-up another.

\section{Conclusion}

Global climate change seriously affects environmental quality, human health, and economic activity, and it requires coordinated policies from countries around the world. Central to the threats of climate change is the increasing tendency of global $\mathrm{CO}_{2}$ emissions, which constitute the single most important greenhouse gas in the atmosphere with a contribution of $64 \%$ out of all long-lived greenhouse gases since 1750. The 2012 greenhouse gas bulletin of the World Meteorological Organization (WMO) documents a 140\% increase in carbon emissions, compared to their level before the industrial era. In Europe, the European Commission has undertaken, since the early-1990s, some initiatives to reduce carbon emissions and combat the consequences of climate change, mainly through the European Climate Change Programme (ECCP). These initiatives include, among others, the establishment of the EU ETS for companies to trade out their emission allowances, proposals on the taxation of energy products, and policy measures to promote electricity from renewable energy and voluntary commitments by car makers to reduce $\mathrm{CO}_{2}$ emissions. This framework requires a comprehensive understanding of the behavior of carbon prices under the effects of not only energy prices but also the prices of electricity produced with different energy sources.

In this study, we have made use of a vine copula approach to investigate the multivariate dependence patterns between carbon prices and energy prices, including electricity prices. The integration of the electricity prices into the analysis is particularly relevant because they influence the choice of power plants and primary fuels, and ultimately carbon emissions and their prices. Moreover, the vine copula-based approach allows to capture a wide range of multidimensional dependence characteristics (i.e., average dependence, upper and lower tail dependence, and asymmetric dependence) of the price variables under consideration for the 
changing market states through time. The effect of the electricity prices is here accounted for by the use a fuel-switching price, which is defined as the $\mathrm{CO}_{2}$ price level for which a gas-fired power plant is as profitable as a coal-fired power plant. We conducted our empirical analysis in three different scenarios to shed light on the role of carbon and electricity prices: i) the dependence structure of energy prices without electricity prices (Case 1); ii) the dependence structure of oil prices, carbon prices, and the fuel-switching price (Case 2); and iii) the dependence structure of all the energy prices (Case 3). For each scenario, we estimate three different multivariate dependence structures with the best copula model for each pair of variables: $\mathrm{C}$-vine, $\mathrm{D}$-vine, and $\mathrm{R}$-vine copulas.

Our results mainly show evidence of a strong and positive link between coal and gas prices, and between coal and oil prices, with or without the presence of electricity prices. However, contrary to the centrality of coal prices in the absence of electricity prices (Scenario 1), the dependence structure is much more complicated in the case where electricity prices are included (Scenario 3). Specifically, we find a positive and high dependence between electricity prices and coal, but negative and high between electricity prices and gas prices. This means that a higher electricity price implied by, for example, a higher consumption can cause a decrease in gas prices but an increase in coal prices and thus more carbon emissions. A weak and positive link is also found between Brent and gas prices, which typically reflects the competition between oil and gas in both consumption and production processes. Due to their substitutes, if the price of one energy source increases significantly, then the end consumers and producers will move to another source of energy, causing its price to rise. This finding is particularly apparent during booming economic activities, as reported in Aloui et al. (2014) who use GARCH-based copula approach to investigate the conditional dependence structure between the crude oil and natural gas markets, and also to derive implications for portfolio risk management in extreme economic conditions.

Another important finding is that carbon prices are only weakly associated with energy prices and their link to gas prices is negative. The latter finding is obtained when electricity prices are included in the multivariate dependence analysis and, thus, implies that high $\mathrm{CO}_{2}$ prices reduce the prices of gas thanks to a decrease in demand. It is worth noting that the main findings for dependence structure remain intact when we replace our switch variable by daily spot prices form EPEX spot electricity prices for Germany/Austria.

Finally, our results suggest that high $\mathrm{CO}_{2}$ prices alone are insufficient to push the elec- 
tricity producers to switch from coal-fired plant to gas-fired plant because they are only weakly and negatively associated with the electricity prices proxied by the switch variable. 


\section{References}

[1] Aas, K., C. Czado, Frigessi, A. and H. Bakken, H. 2009. Pair-copula constructions of multiple dependence. Insurance: Mathematics and Economics 44(2), 182-198.

[2] Aatola, P., Ollikainen, M. and Toppinen, A. 2013. Price determination in the EU ETS market: theory and econometric analysis with market fundamentals. Energy Economics 36, 380-395.

[3] Alberola, E. and Chevallier, J. 2009. European carbon prices and banking restrictions: evidence from Phase I (2005-2007). The Energy Journal, 30(3), 51-79.

[4] Alberola, E., Chevallier, J. and Cheze, B. 2008. Price drivers and structural breaks in European carbon prices 2005-2007. Energy Policy, 42, 332-342.

[5] Aloui, R., Ben Aissa, S., Hammoudeh, S., and Nguyen, D.K. 2014. Dependence and extreme dependence of crude oil and natural gas prices with applications to risk management. Energy Economics, 36 (2), $787-797$.

[6] Andrews, D.W.K. 1993. Tests for parameter instability and structural change with unknown change point. Econometrica, 61(4), 821-856.

[7] Andrews, D.W.K. and Ploberger, W. 1994. Optimal tests when a nuisance parameter is present only under the alternative. Econometrica, 62(6), 1383-1414.

[8] Bai, J., and Perron, P. 1998. Estimating and testing linear models with multiple structural changes. Econometrica, 66, 47-78.

[9] Bedford, T. and R. M. Cooke, R. M. 2001. Probability density decomposition for conditionally dependent random variables modeled by vines. Annals of Mathematics and Artificial intelligence 32, 245-268.

[10] Bedford, T. and R. M. Cooke, R. M. 2002. Vines: a new graphical model for dependent random variables. Annals of Statistics 30, 1031-1068.

[11] Bonneuil, N., Boucekkine, R. 2016. Optimal transition to renewable energy with threshold of irreversible pollution. European Journal of Operational Research 248(1), 257-262.

[12] Brechmann, E.C. and Czado, C., 2011. Risk Management with High-Dimensional Vine Copulas: an Analysis of the Euro Stoxx 50. Working Paper, Technische Universitat Munchen.

[13] Chen, Y., Sijm, J., Hobbs, B. F., Lise, W. 2008. Implications of $\mathrm{CO}_{2}$ emissions trading for short-run electricity market outcomes in northwest Europe. Journal of Regulatory Economics, 34(3), 251-281.

[14] Chevallier, J., 2011. Econometric Analysis of Carbon Markets: The European Union Emissions Trading Scheme and the Clean Development Mechanism, Springer, Berlin, Germany. 
[15] Chevallier, J., Goutte, S. 2017. Estimation of Lévy-driven Ornstein-Uhlenbeck processes: Application to modeling of $\mathrm{CO}_{2}$ and fuel-switching, Annals of Operations Research, 255(1):169-197.

[16] Convery, F. J., and Redmond, L. 2007. Market and price developments in the European Union emissions trading scheme. Review of Environmental Economics and Policy, 1(1), 88-111.

[17] Delarue, E., Ellerman, A.D. and D'haeseleer, W.D. 2009. Short-term $\mathrm{CO}_{2}$ abatement in the European power sector. Climate Change Economics 1(2), 1-21.

[18] Delarue, E. D., Ellerman, A. D., D'haeseleer, W. D. 2010. Robust MACCs? The topography of abatement by fuel switching in the European power sector. Energy, 35(3), 1465-1475.

[19] Delarue, E. and D'haeseleer, W. 2007. Price determination of ETS allowances through the switching level of coal and gas in the power sector. International Journal of Energy Research 31, 1001-1015.

[20] Delarue, E. and D'haeseleer, W. 2008. Greenhouse gas emission reduction-potential by means of fuelswitching in electricity generation: addressing the potentials. Energy Conversion and Management 49, 843-853.

[21] Delarue, E., Voorspools, K. and D'haeseleer, W. 2008. Fuel switching in the electricity sector under the EU ETS: review and prospective. Journal of Energy Engineering ASCE 134, 4-46.

[22] Ding, J.Y., Song, S., Wu, C. 2016. Carbon-efficient scheduling of flow shops by multi-objective optimization. European Journal of Operational Research 248(3), 758-771.

[23] Dißmann, J., Brechmann, E.C., Czado, C. and Kurowicka, D. 2012. Selecting and Estimating Regular Vine Copulae and Application to Financial Returns. Working Paper, Technische Universit?t M?nchen.

[24] Ellerman, A.D. and Buchner, B.K. 2007. The European Union emissions trading scheme: origins, allocation, and early results, Review of Environmental Economics and Policy 1, 66-87.

[25] Ellerman, A.D. and Buchner, B.K. 2008. Over-allocation or abatement? A preliminary analysis of the EU ETS based on the 2005-06 emissions data, Environmental and Resource Economics, 41(2), 267-287.

[26] Hammoudeh, S., Nguyen, D.K., Sousa, R.M. 2014. What explain the short-term dynamics of the prices of $\mathrm{CO}_{2}$ emissions? Energy Economics 46, 122-135.

[27] Hansen, B.E. 1997. Approximate asymptotic P values for structural change tests. Journal of Business and Economic Statistics, 15(1), 60-67.

[28] Hobaek Haff, I., 2013. Parameter estimation for pair-copula constructions. Bernoulli 19(2), 462-491.

[29] Hoffert, M. I., Caldeira, K., Jain, A. K., Haites, E. F., Harvey, L. D., Potter, S. D., Wuebbles, D. J. 1998. Energy implications of future stabilization of atmospheric $\mathrm{CO}_{2}$ content. Nature, 395(6705), $881-884$. 
[30] Jaworski, P., Durante, F., Hardle, W.K., Rychlik, T. 2010. Copula theory and its applications, London: Springer.

[31] Ji, Q., Bouri, E., Roubaud, D., \& Shahzad, S. J. H. (2018). Risk spillover between energy and agricultural commodity markets: A dependence-switching CoVaR-copula model. Energy Economics, 75, $14-27$.

[32] Ji, Q., Liu, B. Y., Nehler, H., \& Uddin, G. S. (2018). Uncertainties and extreme risk spillover in the energy markets: A time-varying copula-based CoVaR approach. Energy Economics, 76, 115-126.

[33] Ji, Q., Zhang, D., \& Geng, J. B. (2018). Information linkage, dynamic spillovers in prices and volatility between the carbon and energy markets. Journal of Cleaner Production, 198, 972-978.

[34] Joe, H. 1997. Multivariate Models and Dependence Concepts. London: Chapman \& Hall..

[35] Koch, N. 2014. Dynamic linkages among carbon, energy and financial markets: a smooth transition approach. Applied Economics, 46(7), 715-729.

[36] Kumar, S., Tiwari, A. K., Chauhan, Y., \& Ji, Q. (2018). Dependence structure between the BRICS foreign exchange and stock markets using the dependence-switching copula approach. International Review of Financial Analysis, forthcoming. https://doi.org/10.1016/j.irfa.2018.12.011

[37] Kurowicka, D. and Cooke, R. M. 2006. Uncertainty Analysis with High Dimensional Dependence Modelling. Chichester: John Wiley.

[38] Lise, W., Sijm, J., Hobbs, B. F. 2010. The impact of the EU ETS on prices, profits and emissions in the power sector: simulation results with the COMPETES EU20 model. Environmental and Resource Economics, 47(1), 23-44.

[39] Nikoloulopoulos, A., Joe, H. and Li, H. 2012. Vine copulas with asymmetric tail dependence and applications to financial return data. Computational Statistics and Data Analysis, 56, 3659-3673.

[40] Lutz, B.J., Pigorsch, U. and Rotfuss, W. 2013. Nonlinearity in cap-and-trade systems: the EUA price and its fundamentals. Energy Economics, 40, 222-232.

[41] Morales-Napoles, O., Cooke, R. M. and Kurowicka, D. 2010. About the number of vines and regular vines on n nodes. Submitted for publication..

[42] Reboredo, J. C. (2013). Modeling EU allowances and oil market interdependence: implications for portfolio management. Energy Economics, 36, 471-480.

[43] Reboredo, J. C. (2014). Volatility spillovers between the oil market and the European Union carbon emission market. Economic Modelling, 36, 229-234. 
[44] Sijm, J., Bakker, S., Chen, Y., Harmsen, H.W. and Lise, W. 2005. $\mathrm{CO}_{2}$ price dynamics: the implications of EU emissions trading for the price of electricity. ECN Working Paper \#05-081.

[45] Sklar, A. 1959. Fonctions de repartition n dimensions et leurs marges. Publications de l'Institut Statistique de l'Universite de Paris 8, 229-231.

[46] Uniejewski, B., Weron, R., \& Ziel, F. (2018). Variance stabilizing transformations for electricity spot price forecasting. IEEE Transactions on Power Systems, 33(2), 2219-2229.

[47] Zachmann, G. 2013. A stochastic fuel switching model for electricity prices. Energy Economics 35, $5-13$.

[48] Zhang, D. (2017). Oil shocks and stock markets revisited: measuring connectedness from a global perspective. Energy Economics, 62, 323-333.

[49] Zhang, D., \& Broadstock, D. C. (2018). Global financial crisis and rising connectedness in the international commodity markets. International Review of Financial Analysis, forthcoming. https://doi.org/10.1016/j.irfa.2018.08.003

[50] Zhang, Y-J., Chen, M-Y. 2016. Evaluating the dynamic performance of energy portfolios: empirical evidence from the DEA directional distance function. European Journal of Operational Research, In press, corrected proof. 


\section{Appendix. Vine copula models}

This appendix describes the main features of the three vine copula models we use in our empirical analysis of multivariate dependence: C-vine, D-vine, and R-vine copulas.

A C-vine copula has a multivariate density given by:

$$
\begin{aligned}
f\left(x_{1}, x_{2}, \ldots, x_{d}\right)= & \prod_{k=1}^{d} f_{k}\left(x_{k}\right) \prod_{h=2}^{d} c_{1, h}\left(F_{1}\left(x_{1}\right), F_{h}\left(x_{h}\right)\right) \\
& \prod_{j=2}^{d-1} \prod_{i=1}^{d-j} c_{j, j+1 \mid 1, \ldots, j-1}\left(F\left(x_{j} \mid x_{1}, \ldots x_{j-1}\right), F\left(x_{j+1} \mid x_{1}, \ldots x_{j-1}\right)\right) .
\end{aligned}
$$

where the conditional distribution functions between variables $x_{i}$ and $x_{j}$ can be obtained (see Joe, 1997) as:

$$
F_{i \mid j}\left(x_{i} \mid x_{j}\right)=c_{i \mid j}\left[F_{i}\left(x_{i}\right), F_{j}\left(x_{j}\right)\right]=\frac{\partial C_{i j}\left[F_{i}\left(x_{i}\right), F_{j}\left(x_{j}\right)\right]}{\partial F_{j}\left(x_{j}\right)},
$$

with $c_{i \mid j}$ denoting the conditional distribution of variable $i$ given the variable $j$ with a joint distribution function $C_{i j}$. The dependence structure of this decomposition can be represented graphically as a hierarchical tree structure. C-vine trees have a star structure where the first root node of the first tree $\mathrm{T}_{1}$ considers the dependence of one variable concerning the other variables as represented by the edge connecting the two nodes. This dependence is given by bivariate copulas (reflected in the second term of Eq. (10)). The tree is then expanded in such a way that the edges of the previous tree form the nodes of the successive trees and, thus consider hierarchical conditional dependencies (reflected in the third term of Eq. (10)). In each tree, one variable plays a pivotal role as it shapes the root node, chosen as the node that maximizes the sum of pairwise dependencies (as measured by Kendall's tau) regarding this node.

A D-vine copula has a multivariate density given by:

$$
\begin{aligned}
f\left(x_{1}, x_{2}, \ldots, x_{d}\right)= & \prod_{k=1}^{d} f_{k}\left(x_{k}\right) \prod_{h=1}^{d-1} c_{h, h+1}\left(F_{h}\left(x_{h}\right), F_{h+1}\left(x_{h+1}\right)\right) \\
& \prod_{j=2}^{d-1} \prod_{i=1}^{d-j} c_{i, i+j \mid i+1, \ldots, i+j-1}\left(F\left(x_{i} \mid x_{i+1}, \ldots x_{i+j-1}\right), F\left(x_{i+j} \mid x_{i+1}, \ldots x_{i+j-1}\right)\right) .
\end{aligned}
$$

The specific ordering of the variables in the D-vine construction is, as can be observed, different from that of the $\mathrm{C}$-vine. The first tree $\left(\mathrm{T}_{1}\right)$ models dependence between the first and second variables, the second and third variables, and so on, using bivariate copulas. 
The edges connecting the nodes represent this dependence relationship. The second tree $\left(\mathrm{T}_{2}\right)$ models the conditional dependence between the first and the third variables, given the second variable, between the second and fourth variables, given the third variable, and so on. This process continues through different trees to the last tree, where only one bivariate dependency remains. We thus have $d(d-1) / 2$ pair copula models. The ordering of the variables in the first tree is crucial because it fully determines the dependence structure of the remaining trees in the D-vine. From $d ! / 2$ possible orderings, the best ordering is selected to capture as much dependence as possible (as measured by Kendall's tau) in the first tree (see Nikoloulopoulos et al. 2012).

According to Kurowicka and Cooke (2006), an R-vine copula consists of linked trees $\mathrm{T}_{1}, \ldots, \mathrm{T}_{d-1}$ with nodes $N_{i}$ and edges $E_{i}$ for $i=1, \ldots, d-1$. The first tree has $d$ nodes and $E_{1}$ edges, whereas each tree of the remaining trees, $i=2, \ldots, d-1, \mathrm{~T}_{i}$ has nodes $N_{i}=E_{i-1}$.

Besides, two edges in tree $\mathrm{T}_{i}$ are joined in tree $\mathrm{T}_{i}+1$ if they share a common node in tree $\mathrm{T}_{i}$ (proximity condition). The edges in an $\mathrm{R}$-vine tree are identified by conditioned nodes denoted by $e=j(e), k(e) \mid D(e)$, where $D(e)$ is the conditioning set. Accordingly, the multivariate density of the R-vine copula is given by

$$
f\left(x_{1}, x_{2}, \ldots, x_{d}\right)=\prod_{k=1}^{d} f_{k}\left(x_{k}\right) \prod_{i=1}^{d-1} \prod_{\epsilon \in E_{i}} c_{j(e), k(e) \mid D(e)}\left(F\left(x_{j(e)} \mid x_{D(e)}\right), F\left(x_{k(e)} \mid x_{D(e)}\right)\right) .
$$

where $x_{D(e)}$ denotes the sub-vector of $x=\left(x_{1}, \ldots, x_{d}\right)$ indicated by the indices contained in $D(e)$.

The $\mathrm{C}$-vine is a special case of the $\mathrm{R}$-vine when each tree has a unique node with degree $d-i$ (the root node). As shown in Morales-Napoloes et al. (2010), there are many different R-vines. To select the most appropriate R-vine, the structure of the tree is selected using the maximum spanning tree that solves the following optimization problem for each tree:

$$
\max \sum_{\text {edges }} \sum_{e=\{i, j\}}\left|\tau_{i j}\right|,
$$

where $\tau_{i, j}$ denotes the pairwise empirical Kendall's tau and a spanning tree is a tree on all nodes. 
Table 1: Descriptive statistics for price returns.

\begin{tabular}{lccccr}
\hline & EUA & Brent & Gas & Coal & Switch \\
\hline \hline Mean & 0.0001 & -0.0001 & 0.0015 & 0.0002 & -0.0023 \\
Std. Dev. & 0.0288 & 0.0173 & 0.0212 & 0.0101 & 0.2664 \\
Max. & 0.2279 & 0.1061 & 0.1695 & 0.0728 & 1.7781 \\
Min. & -0.1186 & -0.0633 & -0.0942 & -0.0837 & -1.7269 \\
Skewness & 0.3143 & 0.3955 & 1.2901 & 0.0952 & -0.3450 \\
Kurtosis & 7.9177 & 6.1943 & 11.5108 & 18.0435 & 17.9146 \\
JB & $1636.6 *$ & $721.1 *$ & $5266.2 *$ & $15070.7 *$ & $14842.1 *$ \\
Q(20) & 22.02 & 16.08 & 25.64 & $52.73 *$ & $53.93 *$ \\
ARCH-LM & $8.32 *$ & $10.27 *$ & $2.30 *$ & $4.82 *$ & $14.10 *$ \\
\hline
\end{tabular}

Notes: Daily data for the period 1 January 2010 to 19 May 2016. JB is the statistic for the test of normality. $\mathrm{Q}(20)$ is the Ljung-Box statistics for serial correlation in the squared returns computed with 20 lags. ARCH-LM is Engle's LM test for heteroskedasticity, computed using 20 lags. An asterisk $(*)$ indicates rejection of the null hypothesis at the $5 \%$ level.

Table 2: Pearson correlation matrix.

\begin{tabular}{lccccc}
\hline & EUA & Brent & Gas & Coal & Switch \\
\hline \hline EUA & 1 & & & & \\
Brent & 0.04 & 1 & & & \\
Gas & 0.02 & 0.11 & 1 & & \\
Coal & 0.03 & 0.15 & 0.25 & 1 & \\
Switch & -0.02 & -0.01 & -0.21 & 0.09 & 1 \\
\hline
\end{tabular}


Table 3: Copula models.

\begin{tabular}{|c|c|c|c|c|c|}
\hline \multirow[b]{2}{*}{ Copula family } & \multicolumn{5}{|c|}{ Dependence features } \\
\hline & positive & negative & symmetric & Lower tail & Upper tail \\
\hline Gaussian (N) & $\checkmark$ & $\checkmark$ & $\checkmark$ & - & - \\
\hline Student-t (t) & $\checkmark$ & $\checkmark$ & $\checkmark$ & $\checkmark$ & $\checkmark$ \\
\hline Clayton (Cl) & $\checkmark$ & - & - & $\checkmark$ & - \\
\hline Gumbel (Gu) & $\checkmark$ & - & - & - & $\checkmark$ \\
\hline Frank $(\mathrm{F})$ & $\checkmark$ & $\checkmark$ & $\checkmark$ & - & - \\
\hline Joe $(\mathrm{J})$ & $\checkmark$ & - & - & - & $\checkmark$ \\
\hline BB1 & $\checkmark$ & - & - & $\checkmark$ & $\checkmark$ \\
\hline BB6 & $\checkmark$ & - & - & $\checkmark$ & $\checkmark$ \\
\hline BB7 & $\checkmark$ & - & - & $\checkmark$ & $\checkmark$ \\
\hline BB8 & $\checkmark$ & - & - & $\checkmark$ & $\checkmark$ \\
\hline
\end{tabular}


Table 4: Parameter estimates for the marginal distribution models.

\begin{tabular}{lccccr}
\hline & EUA & Brent & Gas & Coal & Switch \\
\hline \hline Mean equation & & & & & \\
$\phi_{0}$ & 0.000 & -0.000 & -0.000 & -0.000 & -0.001 \\
& $(0.38)$ & $(0.75)$ & $(-1.83)$ & $(-1.01)$ & $(0.03)$ \\
$\phi_{1}$ & & & & $0.112 *$ & \\
Variance equation & & & & $(5.21)$ & \\
$\omega$ & $0.000 *$ & 0.000 & $0.000 *$ & $0.000 *$ & $0.000 *$ \\
& $(2.49)$ & $(0.77)$ & $(2.44)$ & $(8.62)$ & $(3.67)$ \\
$\alpha_{1}$ & $0.054 *$ & 0.008 & $0.038 *$ & $0.021 *$ & $0.071 *$ \\
& $(3.41)$ & $(1.23)$ & $(3.55)$ & $(6.86)$ & $(2.12)$ \\
$\beta_{1}$ & $0.913 *$ & $0.967 *$ & $0.933 *$ & $0.949 *$ & $0.761 *$ \\
& $(67.74)$ & $(130.67)$ & $(77.96)$ & $(69.61)$ & $(40.88)$ \\
$\lambda$ & $0.052 *$ & $0.050 *$ & $0.050 *$ & $-0.021 *$ & $0.670 *$ \\
& $(2.51)$ & $(4.80)$ & $(2.31)$ & $(-6.44)$ & $(4.95)$ \\
Tail & $5.712 *$ & $7.946 *$ & $4.780 *$ & $2.130 *$ & $2.887 *$ \\
& $(7.26)$ & $(4.73)$ & $(8.88)$ & $(12.46)$ & $(11.65)$ \\
Log-Likelihood & 3658.25 & 4440.58 & 4189.28 & 5181.01 & 1408.41 \\
Q(20) & 21.95 & 12.18 & 18.79 & 23.94 & 22.08 \\
& {$[0.34]$} & {$[0.91]$} & {$[0.53]$} & {$[0.24]$} & {$[0.33]$} \\
Q(20)2 & 13.41 & 15.62 & 10.20 & 27.76 & 7.84 \\
& {$[0.85]$} & {$[0.74]$} & {$[0.96]$} & {$[0.12]$} & {$[0.99]$} \\
ARCH(20) & 0.74 & 0.77 & 0.49 & 1.35 & 0.42 \\
& {$[0.77]$} & {$[0.76]$} & {$[0.96]$} & {$[0.13]$} & {$[0.98]$} \\
K-S & {$[0.85]$} & {$[0.21]$} & {$[0.47]$} & {$[0.36]$} & {$[0.12]$} \\
C-vM & {$[0.91]$} & {$[0.25]$} & {$[0.55]$} & {$[0.34]$} & {$[0.11]$} \\
A-D & {$[0.93]$} & {$[0.38]$} & {$[0.23]$} & {$[0.25]$} & {$[0.11]$} \\
\hline
\end{tabular}

Notes: This table reports the ML estimates and z statistic (in brackets) for the parameters of the marginal distribution models defined in Eqs. (8)- (9). The lags p, q, $r$ and $m$ were selected using the AIC for different combinations of values ranging from 0 to 4 . $\mathrm{Q}(20)$ is the Ljung-Box statistic for serial correlation in the model residuals computed with 20 lags. Q(20)2 represents the LjungBox statistics for serial correlation in the squared residual model calculated with 20 lags. ARCH is the Engle's LM test for the ARCH effect in the residuals up to 20th order. K-S, C-vM and A-D denote the Kolmogorov-Smirnov, Cramér-von Mises and Anderson-Darling test for adequacy of the distribution model. $P$-value (in square parenthesis) below 0.05 indicates rejection of the null hypothesis. Asterisk $(*)$ indicates the significance at $5 \%$. 
Table 5: Case 1: Selection criterion for vine copula models.

\begin{tabular}{lccc}
\hline & C-vine & D-Vine & R-Vine \\
\hline \hline Loglikelihood & 92.67 & 90.52 & 92.31 \\
AIC & -173.35 & -169.04 & -172.62 \\
BIC & -141.09 & -136.79 & -140.36 \\
\hline
\end{tabular}

Table 6: Case 2: Selection criterion for vine copula models.

\begin{tabular}{lccc}
\hline & C-vine & D-Vine & R-Vine \\
\hline \hline Loglikelihood & 2.91 & 2.91 & 2.91 \\
AIC & 0.18 & 0.18 & 0.18 \\
BIC & 16.31 & 16.31 & 16.31 \\
\hline
\end{tabular}

Table 7: Case 3: Selection criterion for vine copula models.

\begin{tabular}{lccc}
\hline & C-vine & D-Vine & R-Vine \\
\hline \hline Loglikelihood & 1172.24 & 1173.05 & 1173.05 \\
AIC & -2318.48 & -2320.11 & -2320.11 \\
BIC & -2248.58 & -2320.11 & -2320.11 \\
\hline
\end{tabular}

